\title{
The Tumor Control According to Radiation Dose of Gamma Knife Radiosurgery for Small and Medium-Sized Brain Metastases from Non-Small Cell Lung Cancer
}

\author{
Sue Jee Park, Sa-Hoe Lim, ${ }^{1}$ Young-Jin Kim, ${ }^{1}$ Kyung-Sub Moon, ${ }^{1}$ In-Young Kim, ${ }^{1}$ Shin Jung, ${ }^{1}$ Seul-Kee Kim, ${ }^{2}$ In-Jae Oh, ${ }^{3}$ \\ Jong-Hwan Hong, ${ }^{1}$ Tae-Young Jung ${ }^{1}$ \\ Department of Neurosurgery, Chonnam National University Hwasun Hospital, Chonnam National University Medical School, Hwasun, Korea \\ Department of Radiology, ${ }^{2}$ Chonnam National University Hwasun Hospital, Chonnam National University Medical School, Hwasun, Korea \\ Department of Internal Medicine, ${ }^{3}$ Chonnam National University Hwasun Hospital, Chonnam National University Medical School, Hwasun, Korea
}

Objective : The effectiveness of gamma knife radiosurgery (GKR) in the treatment of brain metastases is well established. The aim of this study was to evaluate the efficacy and safety of maximizing the radiation dose in GKR and the factors influencing tumor control in cases of small and medium-sized brain metastases from non-small cell lung cancer (NSCLC).

Methods: We analyzed 230 metastatic brain tumors less than $5 \mathrm{~mL}$ in volume in 146 patients with NSCLC who underwent GKR. The patients had no previous radiation therapy for brain metastases. The pathologies of the tumors were adenocarcinoma $(n=207)$, squamous cell carcinoma ( $n=18)$, and others $(n=5)$. The radiation doses were classified as $18,20,22$, and $24 \mathrm{~Gy}$, and based on the tumor volume, the tumors were categorized as follows : small-sized (less than $1 \mathrm{~mL}$ ) and medium-sized (1-3 and 3-5 mL). The progression-free survival (PFS) of the individual 230 tumors and 146 brain metastases was evaluated after GKR depending on the pathology, Eastern Cooperative Oncology Group (ECOG) performance score (PS), tumor volume, radiation dose, and anti-cancer regimens. The radiotoxicity after GKR was also evaluated.

Results : After GKR, the restricted mean PFS of individual 230 tumors at 24 months was 15.6 months (14.0-17.1). In small-sized tumors, as the dose of radiation increased, the tumor control rates tended to increase ( $p=0.072)$. In medium-sized tumors, there was no statistically difference in PFS with an increase of radiation dose $(p=0.783)$. On univariate analyses, a statistically significant increase in PFS was associated with adenocarcinomas ( $p=0.001)$, tumors with ECOG PS $0(p=0.005)$, small-sized tumors $(p=0.003)$, radiation dose of $24 \mathrm{~Gy}(p=0.014)$, synchronous lesions $(p=0.002)$, and targeted therapy $(p=0.004)$. On multivariate analyses, an improved PFS was seen with targeted therapy (hazard ratio, $0.356 ; 95 \%$ confidence interval, $0.150-0.842 ; p=0.019$ ). After GKR, the restricted mean PFS of brain at 24 months was 9.8 months (8.5-11.1) in 146 patients, and the pattern of recurrence was mostly distant within the brain (66.4\%). The small and medium-sized tumors treated with GKR showed radiotoxicitiy in five out of 230 tumors (2.2\%), which were controlled with medical treatment.

Conclusion : The small-sized tumors were effectively controlled without symptomatic radiation necrosis as the radiation dose was increased up to $24 \mathrm{~Gy}$. The medium-sized tumors showed potential for symptomatic radiation necrosis without signifcant tumor control rate, when greater than $18 \mathrm{~Gy}$. GKR combined targeted therapy improved the tumor control of GKR-treated tumors.

Key Words : Radiosurgery · Radiation · Neoplasm metastasis · Radiation injuries · Drug therapy.

- Received : July 5, $2021 ・$ Revised : August 30, $2021 ・$ Accepted : September 7, 2021

- Address for reprints : Tae-Young Jung

Department of Neurosurgery, Chonnam National University Hwasun Hospital, 322 Seoyang-ro, Hwasun 58128, Korea

Tel : +82-61-379-7670, Fax : +82-61-379-7673, E-mail : jung-ty@chonnam.ac.kr, ORCID : https://orcid.org/0000-0002-8685-8520

\section{In-Jae Oh}

Department of Internal Medicine, Chonnam National University Hwasun Hospital, 322 Seoyang-ro, Hwasun 58128, Korea

Tel : +82-61-379-7617, Fax: +82-61-379-7619, E-mail : droij@chonnam.ac.kr, ORCID : https://orcid.org/0000-0003-4837-1321

This is an Open Access article distributed under the terms of the Creative Commons Attribution Non-Commercial License (http://creativecommons.org/licenses/by-nc/4.0) which permits unrestricted non-commercial use, distribution, and reproduction in any medium, provided the original work is properly cited. 


\section{INTRODUCTION}

Brain metastases are found in about $10-20 \%$ of patients with non-small cell lung cancer (NSCLC) at the time of the first diagnosis, and approximately $40 \%$ of all patients develop brain metastases during the course of their disease $\mathrm{e}^{1)}$. As the survival rate of patients with NSCLC has increased, brain metastases are being diagnosed with increasing frequency and are becoming a major cause of morbidity and mortality ${ }^{8}$. The treatment options for brain metastases include surgical resection, whole brain radiation therapy (WBRT), gamma knife radiosurgery (GKR), and combinations of these ${ }^{8,15}$. In recent times, GKR has been described as an effective treatment for small and medium-sized metastatic tumors, considering the minimal invasiveness of GKR and lower risk of adverse effects compared with $\mathrm{WBRT}^{8,29)}$.

For GKR, radiation doses are determined according to the tumor volume, history of previous brain treatment, and the pathology of primary cancer. A higher radiation dose is associated with more radiation toxicity, such as radiation necrosis, while a low dose might result in poor tumor control ${ }^{2,8}$. Given that irradiation for large-sized tumors has been associated with radiation necrosis, the radiation dose based on tumor volume is a critical factor in GKR that influences outcomes ${ }^{8,10,30}$. Cytotoxic chemotherapeutic agents cannot efficiently penetrate the blood-brain barrier (BBB). Thus, it has a limitation for the treatment of brain metastases. However, some recent molecular targeted therapies have shown good results in treating brain metastases, with a significant improvement in overall survival $(\mathrm{OS})^{1,28)}$. Therefore, GKR combined the anti-cancer regimen also affects tumor control.

There have been many questions about whether tumor control increases by maximizing the radiation dose and which prognostic factors, including radiation dose and anti-cancer regimen, influence tumor control. Therefore, the aim of this study was to evaluate the efficacy and safety of maximizing the radiation dose of GKR and the factors influencing tumor control in cases of small and medium-sized brain metastases from NSCLC.

\section{MATERIALS AND METHODS}

This retrospective study was approved by the Institutional
Review Board of Chonnam National University Hwasun Hospital (CNUHH-2021-133), and the need for written informed consent was waived because of the retrospective design of this study.

\section{Clinical characteristics of $\mathbf{1 4 6}$ patients with NSCLC}

From January 2012 to December 2019, 746 patients with a total of 3363 metastatic brain tumors from NSCLC were treated with GKR. The inclusion criteria were as follows : number of tumors less than 4, tumor volume less than $5 \mathrm{~mL}$, Eastern Cooperative Oncology Group (ECOG) performance status (PS) grade 2 or less, no previous history of radiation therapy for brain metastases, and availability of clinical data and data from radiologic follow-up for more than 3 months. Based on the inclusion criteria, a total of 146 patients with 230 tumors were included in this study.

The characteristics of the 146 patients are summarized in Table 1. The male-to-female ratio was $97: 49$, and the median age was 64 years (range, 33-85). The pathological types of NSCLC were adenocarcinoma ( $\mathrm{n}=130,89 \%)$, squamous cell carcinoma $(n=14,9.6 \%)$, and others $(n=2,1.4 \%)$. Other pathologies were neuroendocrine carcinoma and NSCLC not otherwise specified. The ECOG PS scale (grades 0-5) was used to assess disease progression and how the disease and treatments affected the activities of daily living, to determine the appropriate treatment, and for estimating prognosis ${ }^{3)}$. The number of patients with ECOG PS grade 0 was 74 (50.7\%), grade 1 was 65 (44.5\%), and grade 2 was 7 (4.8\%). The Radiation Therapy Oncology Group (RTOG) prognostic classes for brain metastases, which was based on a recursive partitioning analysis (RPA) and utilizes a three-class system for the stratification and reporting of brain metastases, was used to classify the patients. The classification is as follows : class I : Karnofsky performance status (KPS) $\geq 70$, less than 65 years of age with controlled primary, and no extracranial metastases; class III : KPS $<70$; class II : all others ${ }^{9)}$. The number of patients with RPA class I and RPA class II was 26 (17.8\%) and 120 (82.2\%), respectively. Ninety patients (61.6\%) had synchronous lesions, and 56 (38.4\%) had metachronous lesion. The tumor control effect based on the anti-cancer regimens used after GKR was analyzed. The number of patients who received cytotoxic systemic chemotherapy for NSCLC after GKR was 76 (52.1\%), and 51 patients $(34.9 \%)$ received molecular targeted therapy. Best supportive care (BSC) without chemotherapy was pro- 
Table 1. Sample characterization of 146 patients and 230 GKR treated lesions

\begin{tabular}{|c|c|c|}
\hline Clinical factor & $\begin{array}{l}\text { Patients } \\
(n=146)\end{array}$ & $\begin{array}{l}\text { Tumors } \\
(n=230)\end{array}$ \\
\hline Age (years) & $64(33-85)$ & \\
\hline Male to female ratio & $97: 49$ & \\
\hline Number of lesion/person & & $1(1-3)$ \\
\hline Tumor volume (mL) & & $0.3839(0.0085-4.9700)$ \\
\hline \multicolumn{3}{|l|}{ Pathology } \\
\hline Adenocarcinoma & $130(89.0)$ & $207(90.0)$ \\
\hline Squamous cell carcinoma & $14(9.6)$ & $18(7.8)$ \\
\hline Others & $2(1.4)$ & $5(2.2)$ \\
\hline
\end{tabular}

$\begin{array}{lrr}\text { Time interval of brain metastasis } & & \\ \text { Synchronous } & 90(61.6) & 147(63.9) \\ \text { Metachronous } & 56(38.4) & 83(36.1)\end{array}$

RPA class

\begin{tabular}{ccc} 
Class I & $26(17.8)$ & $41(17.8)$ \\
Class II & $120(82.2)$ & $189(82.2)$ \\
\hline ECOGPS & & \\
0 & $74(50.7)$ & $115(50.0)$ \\
1 & $65(44.5)$ & $102(44.3)$ \\
2 & $7(4.8)$ & $13(5.7)$
\end{tabular}

\section{Tumor volume $(\mathrm{mL})$}

$\begin{array}{ll}<1 & 162(70.4) \\ 1-3 & 47(20.4) \\ 3-5 & 21(9.1)\end{array}$

\section{Radiation dose}

$24 \mathrm{~Gy} \quad 35(15.2)$

$22 \mathrm{~Gy} \quad 91(39.6)$

20 Gy $\quad 93(40.4)$

$18 \mathrm{~Gy} \quad 11(4.8)$

Anti-cancer regimen

$\begin{array}{lll}\text { Targeted therapy } & 51(34.9) & 89(38.7) \\ \text { Cytotoxic chemotherapy } & 76(52.1) & 16(50.4) \\ \text { Best supportive care } & 19(13.0) & 25(10.9)\end{array}$

Recurrence pattern in the brain

$\begin{array}{lc}\text { No recurrence } & 44(30.1) \\ \text { Local } & 3(2.1) \\ \text { Distant including local } & 97(66.4) \\ \text { CSF seeding } & 2(1.4)\end{array}$

Values are presented as median (range) or number (\%). GKR : gamma knife radiosurgery, RPA : recursive partitioning analysis, ECOG: European Cooperative Oncology Group, PS : performance score, CSF : cerebrospinal fluid vided to 19 patients (13.0\%).

After GKR, the patterns of brain recurrence were classified as local, distant, and cerebrospinal fluid (CSF) seeding. Local recurrence was defined as recurrence at the same site as the original GKR-treated lesion and distance recurrence as a metastasis spatially separated from the GKR-treated lesion. CSF seeding was defined as recurrence in the ventricles, subarachnoid space, and spinal cord diagnosed by imaging studies or tumor cells on CSF cytology. Out of 146 patients, 44 patients (30.1\%) showed no recurrence; three $(2.1 \%)$ developed local recurrence; 97 (66.4\%) had distant recurrence; and two (1.4\%) presented with CSF seeding.

\section{GKR for $\mathbf{2 3 0}$ metastatic tumors}

GKR was performed using the Leksell GammaPlan ${ }^{\circledR}$ radiosurgery planning software (Elekta Instruments AB, Stockholm, Sweden) for all 230 individual brain tumors. The characteristics of the 230 tumors are summarized in Table 1. The median number of lesions per patient was 1 (range, 1-3). The median target volume was $0.3839 \mathrm{~mL}$ (range, 0.0085-4.9700). The tumors were classified into small-sized $(<1 \mathrm{~mL}, \mathrm{n}=162$, $70.4 \%$ ) and medium-sized ( $1-3 \mathrm{~mL}, \mathrm{n}=47,20.4 \%$ and $3-5 \mathrm{~mL}$, $\mathrm{n}=21,9.1 \%$ ) according to the tumor volume. The marginal prescription doses ranged from 18 to $24 \mathrm{~Gy}$ (mean, $22 \mathrm{~Gy}$ ). The range of prescription isodose percentage was from $40 \%$ to 80\% (median, 50\%; mean 55.7\%). The radiation doses were divided into 18 Gy ( $\mathrm{n}=11,4.8 \%), 20 \mathrm{~Gy}(\mathrm{n}=93,40.4 \%), 22 \mathrm{~Gy}$ $(\mathrm{n}=91,39.6 \%)$, and $24 \mathrm{~Gy}(\mathrm{n}=35,15.2 \%)$.

Serial contrast-enhanced magnetic resonance imaging (MRI) was performed during the follow-up conducted more than 3 months after the GKR. Recurrence was diagnosed in cases of histological confirmation, or tumor control after additional local treatment with radiologic follow-up. The additional local treatment included repeat GKR, whole-brain radiotherapy, and surgery. In our study, 69 lesions were radiologically diagnosed as recurrence.

As an adverse effect of GKR, radiation necrosis was diagnosed on the basis of pathologic confirmation or radiologic follow-up. Radiologically, the tumor with radiation necrosis was stable, reduced or increased, and the aggravated perilesional edema was improved with or without symptomatic medical treatment. If necessary, methionine-positron emission tomography and diffusion and perfusion-weighted MRI sequences were performed for the diagnosis of radiation ne- 
crosis. In our study, five (2.2\%) out of 230 lesions were radiologically diagnosed as having radiation necrosis.

\section{Statistical analysis}

Variables are presented as the restricted mean progressionfree survival (PFS) instead of the median value because the follow-up period was short with small event rates. The effects of single variables on PFS and OS were determined by univariate and multivariate analyses. The single variables were ECOG PS, radiation dose, tumor volume, tumor pathology, and anticancer regimen. PFS was calculated from the date of GKR to the date of recurrence or last follow-up visit, and OS was calculated from the date of GKR to the date of death or last follow-up. Survival probability was calculated using the KaplanMeier method, and comparisons were made using a log-rank test. We examined variables using a Cox proportional hazard analysis model to identify the independent predictors of survival. A linear regression analysis was performed to assess the relationship between PFS of the GKR-treated lesions and the duration of targeted chemotherapy. All statistical analyses were performed with a significance level of $p<0.05$ using SPSS ver. 21.0 (SPSS, Chicago, IL, USA).

\section{RESULTS}

\section{PFS of 230 GKR-treated lesions}

After GKR, the restricted mean PFS of the 230 tumors at 24 months was 15.6 months (14.0-17.1). The univariate analyses findings are summarized in Table 2 . There was no statistical significance in distribution of RPA class $(p=0.437)$. Depending on the pathologies, the restricted mean PFS at 8 months was 7.5 months (7.3-7.7) for adenocarcinoma, 5.9 months (4.8-6.9) for squamous cell carcinoma and 7.1 months (6.18.0) for others $(p=0.001)$. Depending on the ECOG PS, the restricted mean PFS at 12 months was 11.0 months (10.5-11.5) for PS 0, 10.1 months (7.9-12.4) for PS 1, and 10.0 months (9.3-10.7) for PS 2 ( $p=0.005)$. The restricted mean PFS at 24 months of small-sized tumors with a volume of less than $1 \mathrm{~mL}$ was 20.0 months (18.7-21.2); medium-sized tumors with volume with volume 1-3 mL, 15.7 months (12.8-18.6); and medium-sized tumors with volume $3-5 \mathrm{~mL}$ was 15.0 months (10.8-19.3) ( $p=0.003)$ (Fig. 1A). Depending on the radiation dose, the restricted mean PFS at 24 months at a dose of $24 \mathrm{~Gy}$ was 21.8 months (19.5-24.1); $22 \mathrm{~Gy}, 19.7$ months (17.9-21.6); 20 Gy, 17.5 months (15.7-19.4); and 18 Gy, 13.7 months (8.7-18.6) $(p=0.014)$ (Fig. 1B). The restricted mean PFS of synchronous

Table 2. Progression-free survival of 230 GKR-treated lesions and 146 brains, and overall survival of 146 patients

\begin{tabular}{|c|c|c|c|c|c|}
\hline \multirow{2}{*}{ Clinical factor } & \multirow{2}{*}{$\begin{array}{l}\text { Restricted mean PFS } \\
(95 \% \mathrm{CI})\end{array}$} & \multirow{2}{*}{$p$-value } & \multirow{2}{*}{$\frac{\text { Brain PFS }}{p \text {-value }}$} & \multirow{2}{*}{$\begin{array}{l}\text { Restricted mean OS } \\
\qquad(95 \% \mathrm{CI})\end{array}$} & \multirow{2}{*}{$\begin{array}{c}\text { OS } \\
p \text {-value }\end{array}$} \\
\hline & & & & & \\
\hline \multicolumn{6}{|c|}{$\begin{array}{l}\text { Univariate analysis related with PFS of GKR-treated lesions } \\
(\mathrm{n}=230)\end{array}$} \\
\hline Pathology & & 0.001 & & & \\
\hline Adenocarcinoma & $7.5(7.3-7.7)$ & & & & \\
\hline Squamous cell carcinoma & $5.9(4.8-6.9)$ & & & & \\
\hline Others & $7.1(6.1-8.0)$ & & & & \\
\hline Time interval of brain metastasis & & 0.002 & & & \\
\hline Synchronous & $20.0(18.7-21.2)$ & & & & \\
\hline Metachronous & $16.1(13.8-18.5)$ & & & & \\
\hline RPA class & & 0.437 & & & \\
\hline Class 1 & $18.8(17.5-20.1)$ & & & & \\
\hline Class $\|$ & $17.7(14.8-20.6)$ & & & & \\
\hline ECOG PS & & 0.005 & & & \\
\hline 0 & $11.0(10.5-11.5)$ & & & & \\
\hline 1 & $10.1(7.9-12.4)$ & & & & \\
\hline 2 & $10.0(9.3-10.7)$ & & & & \\
\hline
\end{tabular}


The Tumor Control after GKR I Park SJ, et al.

Table 2. Continued

\begin{tabular}{|c|c|c|c|c|c|}
\hline \multirow{2}{*}{ Clinical factor } & \multirow{2}{*}{$\begin{array}{l}\text { Restricted mean PFS } \\
\qquad(95 \% \mathrm{Cl})\end{array}$} & \multirow{2}{*}{$p$-value } & \multirow{2}{*}{$\frac{\text { Brain PFS }}{p \text {-value }}$} & \multirow{2}{*}{$\begin{array}{l}\text { Restricted mean OS } \\
\qquad(95 \% \mathrm{Cl})\end{array}$} & \multirow{2}{*}{$\frac{\text { OS }}{p \text {-value }}$} \\
\hline & & & & & \\
\hline Tumor volume $(\mathrm{mL})$ & & 0.003 & & & \\
\hline$<1$ & $20.0(18.7-21.2)$ & & & & \\
\hline $1-3$ & $15.7(12.8-18.6)$ & & & & \\
\hline $3-5$ & $15.0(10.8-19.3)$ & & & & \\
\hline Radiation dose & & 0.014 & & & \\
\hline 24 Gy & $21.8(19.5-24.1)$ & & & & \\
\hline $22 \mathrm{~Gy}$ & $19.7(17.9-21.6)$ & & & & \\
\hline 20 Gy & $17.5(15.7-19.4)$ & & & & \\
\hline 18 Gy & $13.7(8.7-18.6)$ & & & & \\
\hline Anti-cancer regimen & & 0.004 & & & \\
\hline Targeted therapy & $20.8(19.2-22.3)$ & & & & \\
\hline Cytotoxic chemotherapy & $17.6(15.8-19.3)$ & & & & \\
\hline Best supportive care & $14.8(10.0-19.5)$ & & & & \\
\hline \multicolumn{6}{|c|}{$\begin{array}{l}\text { Univariate analysis related with brain PFS and OS of } \\
\text { patients ( } n=146)\end{array}$} \\
\hline Pathology & & & 0.083 & & 0.003 \\
\hline Adenocarcinoma & & & & $8.2(7.9-8.5)$ & \\
\hline Squamous cell carcinoma & & & & $8.1(6.8-9.3)$ & \\
\hline Others & & & & $7.5(6.7-8.4)$ & \\
\hline Time interval of brain metastasis & & & 0.163 & & 0.310 \\
\hline Synchronous & & & & $16.3(14.7-17.9)$ & \\
\hline Metachrnous & & & & $14.9(12.9-17.0)$ & \\
\hline RPA class & & & 0.640 & & 0.085 \\
\hline Class 1 & & & & $18.2(15.5-20.8)$ & \\
\hline Class II & & & & $15.3(13.8-16.7)$ & \\
\hline ECOG PS & & & 0.084 & & 0.910 \\
\hline 0 & & & & $15.8(14.1-17.5)$ & \\
\hline 1 & & & & $16.2(14.2-18.1)$ & \\
\hline 2 & & & & $12.3(5.6-18.9)$ & \\
\hline Anti-cancer regimen & & & 0.393 & & 0.012 \\
\hline Targeted therapy & & & & $18.3(16.3-20.4)$ & \\
\hline Cytotoxic chemotherapy & & & & $14.9(13.2-16.6)$ & \\
\hline Best supportive care & & & & $12.4(8.9-15.9)$ & \\
\hline Recurrene pattern in the brain & & & & & 0.072 \\
\hline No recurrence & & & & $9.1(8.1-10.1)$ & \\
\hline Local & & & & $12.0(12.0-12.0)$ & \\
\hline Distant including local & & & & $10.6(10.1-11.1)$ & \\
\hline CSF seeding & & & & $8.9(4.6-13.1)$ & \\
\hline
\end{tabular}

GKR: gamma knife radiosurgery, PFS : progression-free survival, Cl : confidence interval, OS : overall survival, RPA : recursive partitioning analysis, ECOG : European Cooperative Oncology Group, PS : performance score, CSF : cerebrospinal fluid 
brain tumors at 24 months was 20.0 months (18.7-21.2), and for metachronous tumors, it was 16.1 months (13.8-18.5) $(p=0.002)$. Depending on the anti-cancer regimens, the restricted mean PFS of targeted therapy at 24 months was 20.8 months (19.2-22.3); for cytotoxic chemotherapy, 17.6 months (15.8-19.3); and for BSC, 14.8 months (10.0-19.5) $(p=0.004)$ (Fig. 1C). Particularly for lesions treated that received GKR combined targeted therapy, there was a correlation between PFS and duration of targeted therapy ( $\mathrm{\gamma}=0.640, p=0.001$ ) (Fig. 1D).

After classifying the tumor volume as less than $1 \mathrm{~mL}$ and 1 $\mathrm{mL}$ or more, the PFS of the tumors according to each radiation dose was analyzed. For tumors with volumes less than 1 $\mathrm{mL}$, there was no statistically significant difference in PFS, but it tended to increase as the radiation dose increased (Fig. 2A, $p=0.072$ ). For small-sized tumors with volume less than $1 \mathrm{~mL}$, the restricted mean PFS at 24 months was 21.9 months (19.724.1) for a dose of $24 \mathrm{~Gy}, 20.9$ months (19.1-22.8) for $22 \mathrm{~Gy}$, and 18.5 months (16.4-20.7) for 20 Gy. For medium-sized tumors with volume more than $1 \mathrm{~mL}$ and less than $5 \mathrm{~mL}$, the restricted mean PFS at 24 months was 16.6 months (12.5-20.8) for a dose of $22 \mathrm{~Gy}, 15.8$ months (12.3-19.3) for $20 \mathrm{~Gy}$, and 13.7 months (8.7-18.6) for 18 Gy (Fig. 2B, $p=0.783$ ).

The multivariate analyses findings related with PFS are summarized in Table 3. GKR combined targeted therapy showed improved PFS (hazard ratio, 0.356; 95\% confidence interval [CI], 0.150-0.842; $p=0.019)$ compared to other clinical variables including tumor volume, radiation dose and anticancer regimens.
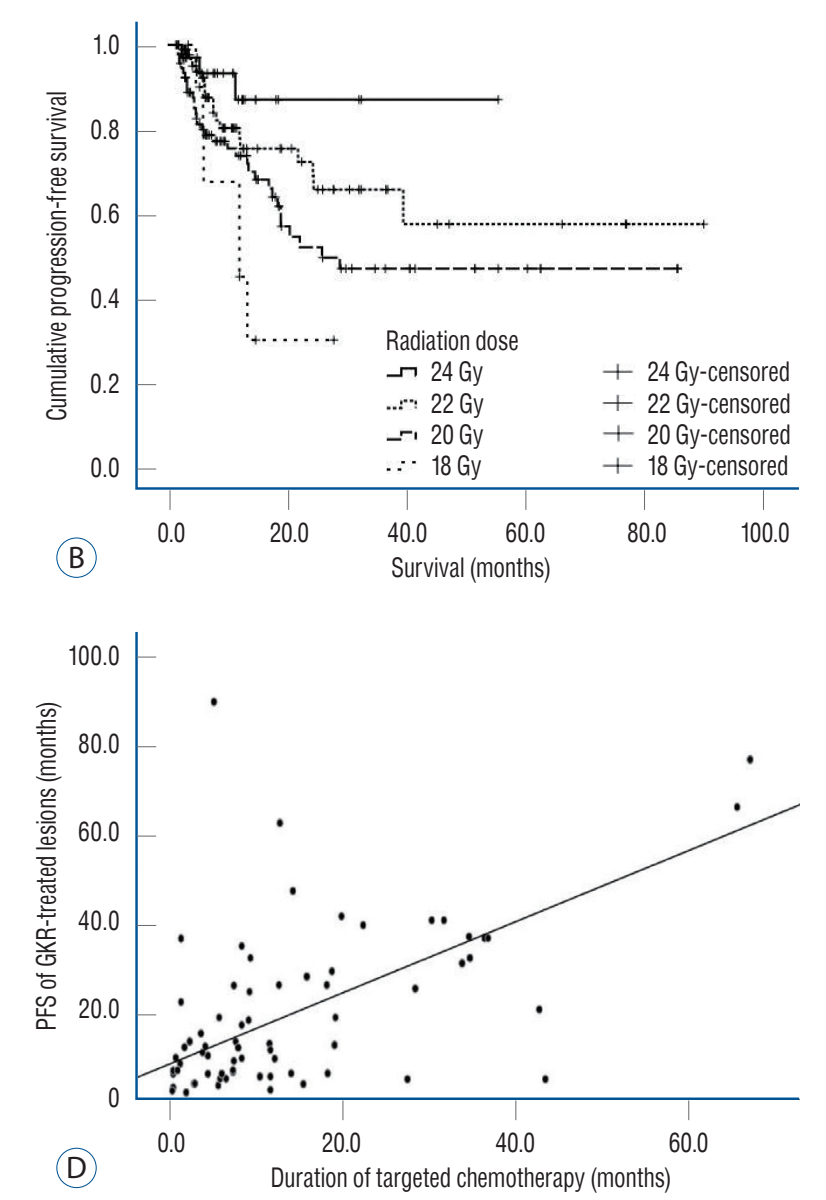
(C)
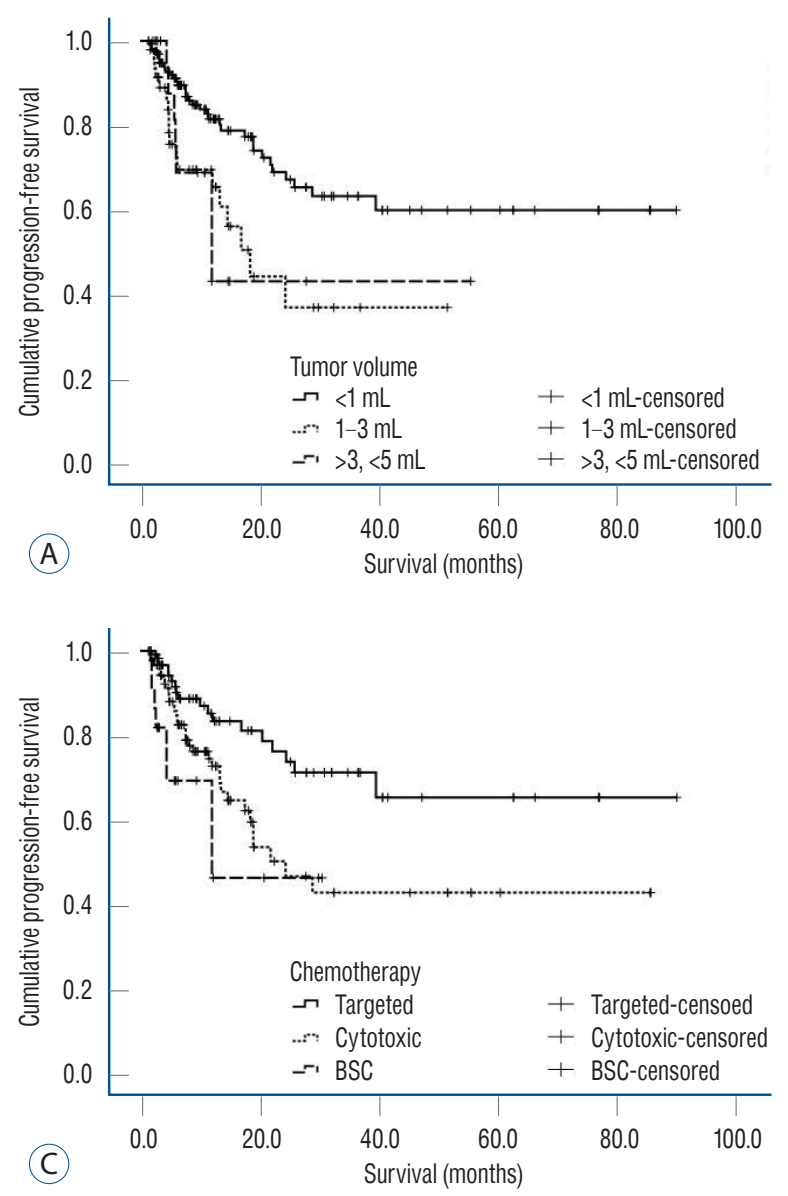

Fig. 1. The progression-free survival of 230 GKR-treated metastatic lesions. A : The small-sized tumors less than $1 \mathrm{~mL}$ showed an improved PFS than the medium-sized tumors ( $p=0.003)$. B : The marginal radiation dose of $24 \mathrm{~Gy}$ showed an improved PFS than doses of $20 \mathrm{~Gy}$ and $18 \mathrm{~Gy}(p=0.014)$. C : Targeted chemotherapy showed an improved PFS compared to that with cytotoxic chemotherapy or best supportive care $(p=0.004)$. $D:$ There was a correlation between PFS of GKR-treated lesions and duration of targeted chemotherapy after GKR $(\gamma=0.640, p=0.001)$. BSC : best supportive care, GKR : gamma knife radiosurgery, PFS : progression-free survival. 


\section{PFS of brain after GKR and OS of $\mathbf{1 4 6}$ patients}

After GKR, the restricted mean PFS of the brain at 24 months was 9.8 months (8.5-11.1) in 146 patients. The results of the univariate analyses related to PFS are summarized in Table 2. There was no statistical significant effect of pathology $(p=0.083)$, time of brain metastasis $(p=0.163)$, RPA class $(p=0.640)$, ECOG PS $(p=0.084)$, and treatment regimens $(p=0.393)$ on PFS.

After GKR, the restricted mean OS at 24 months was 12.1 months (10.9-13.3) in 146 patients. The results of the univari-
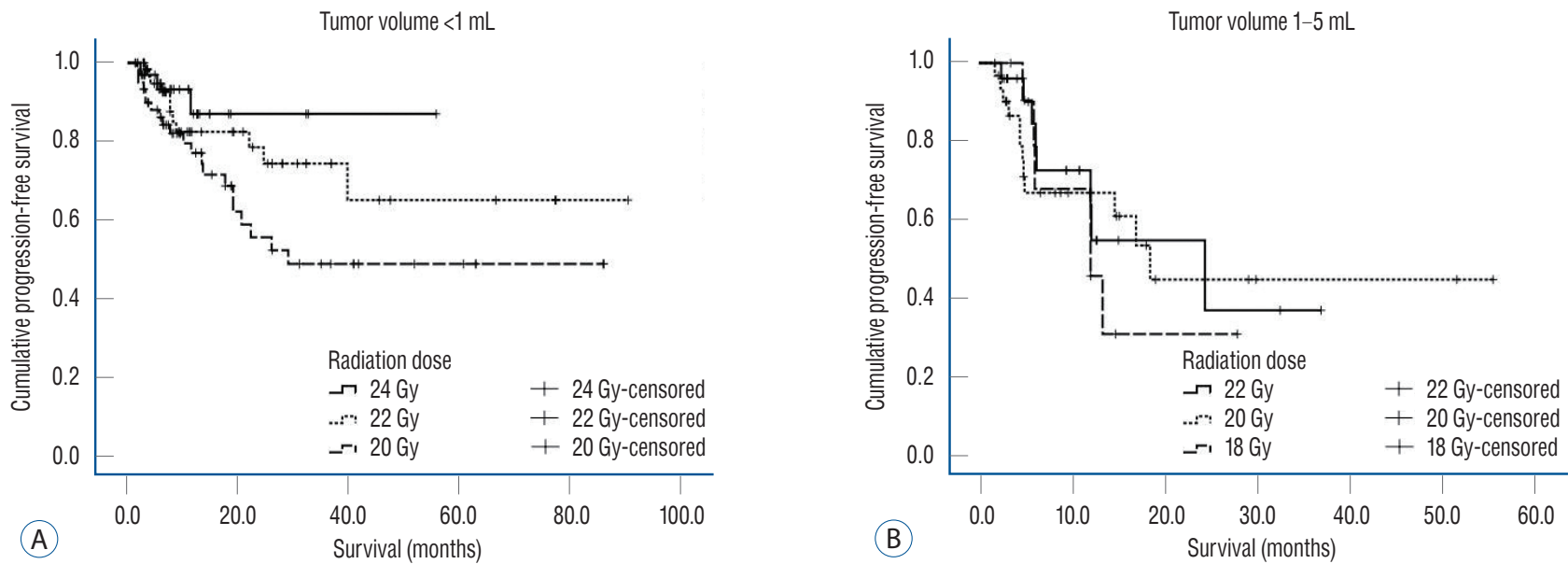

Fig. 2. The progression-free survival of 230 gamma knife radiosurgery-treated lesions depending on the tumor volume and radiation dose. A : For small-sized tumors less than $1 \mathrm{~mL}$, progression-free survival tended to increase with an increase in the radiation dose but without a statistically significant difference ( $p=0.072$ ). B : For medium-sized tumors $>1$ and $<5 \mathrm{~mL}$, there was no statistically significant difference in the outcomes with different radiation doses $(p=0.783)$.

Table 3. Prognostic factors related with progression free survival of GKR treated lesions and overall survival of patients

\begin{tabular}{|c|c|c|c|}
\hline Clinical factor & Hazard ratio & $95 \% \mathrm{Cl}$ & $p$-value \\
\hline \multicolumn{4}{|c|}{ Multivariate analysis related with PFS of GKR-treated lesions ( $n=230$ ) } \\
\hline Tumor volume, $<1 \mathrm{~mL}$ & 0.601 & $0.339-1.066$ & 0.082 \\
\hline \multicolumn{4}{|l|}{ Radiation dose } \\
\hline 24 Gy & 0.277 & $0.061-1.266$ & 0.098 \\
\hline $22 \mathrm{~Gy}$ & 0.554 & $0.203-1.514$ & 0.249 \\
\hline 20 Gy & 0.840 & $0.325-2.173$ & 0.720 \\
\hline \multicolumn{4}{|l|}{ Anti-cancer regimen } \\
\hline Targeted therapy & 0.356 & $0.150-0.842$ & 0.019 \\
\hline Cytotoxic chemotherapy & 0.716 & $0.325-1.577$ & 0.407 \\
\hline \multicolumn{4}{|c|}{ Multivariate analysis related with OS of patients ( $n=146$ ) } \\
\hline \multicolumn{4}{|l|}{ Pathology } \\
\hline Adenocarcinoma & 0.250 & $0.060-1.043$ & 0.057 \\
\hline Squamous cell carcinoma & 0.714 & $0.156-3.279$ & 0.665 \\
\hline RPA class, class I & 0.539 & $0.316-0.920$ & 0.023 \\
\hline \multicolumn{4}{|l|}{ Anti-cancer regimen } \\
\hline Targeted therapy & 0.436 & $0.234-0.813$ & 0.009 \\
\hline Cytotoxic chemotherapy & 0.619 & $0.347-1.103$ & 0.103 \\
\hline
\end{tabular}

Reference variables : 1-5 mL tumor volume, 18 Gy radiation dose, supportive care, other pathology, RPA class II. GKR : gamma knife radiosurgery, Cl : confidence interval, PFS : progression-free survival, OS : overall survival, RPA : recursive partitioning analysis 
ate analyses related to OS are summarized in Table 2. There was no statistical significant effect of the time of metastasis $(p=0.310)$, RPA class $(p=0.085)$, ECOG PS $(p=0.910)$, and recurrence pattern $(p=0.072)$ on OS. Depending on the pathologies, the restricted mean OS at 9 months was 8.2 months (7.98.5) for patients with adenocarcinoma, 8.1 months (6.8-9.3) for those with squamous cell carcinoma, and 7.5 months (6.78.4) for those with other types ( $p=0.003$ ). Depending on the anti-cancer regimens, the restricted mean OS at 24 months of patients who received targeted therapy was 18.3 months (16.320.4); patients who received cytotoxic chemotherapy, 14.9 months (13.2-16.6); and patients who received BSC, 12.4 months (8.9-15.9) $(p=0.012)$. The results of the multivariate analyses related to OS are summarized in Table 3. Out of the clinical factors assessed, which included pathology, RPA class, and anti-cancer regimens, targeted therapy and RPA class I showed improved OS (hazard ratio, 0.436; 95\% CI, 0.234-0.813; $p=0.009$ and hazard ratio, $0.539 ; 95 \% \mathrm{CI}, 0.316-0.920$; $p=0.023$, respectively).

\section{Summary of cases with radiation necrosis after GKR}

Five (2.2\%) out of 230 GKR-treated lesions showed aggravated perilesional edema : three tumor lesions remained stable in size, and two lesions increased in size at a median of 1.7 months (range, 1.4-3.4) after GKR. The median age of patients with radiation necrosis was 64 years (range, 54-72); the male to female was $3: 2$, and the pathology was adenocarcinoma in four and squamous cell carcinoma in one. After GKR, one patient received targeted therapy, two patients received cytotoxic chemotherapy, and two patients were provided BSC without anti-cancer treatment. The median radiation volume was $1.83 \mathrm{~mL}$ (range, 0.7539-4.1100) and the median marginal radiation dose was 20 Gy (range, 18-24). The median percentage of prescription isodose was 50 (range, 50-80). Three patients showed neurological symptoms; of them, two had a headache and one had a seizure; the remaining two patients had no neurological symptoms. Two patients were administered steroids; one patient was administered prednisolone 20 mg a day for a total of 42 days, and the dosage for the other patient was $20 \mathrm{mg}$ a day for 30 days, followed by $10 \mathrm{mg}$ a day for 60 days, and the symptoms of both patients improved. The lesions showed radiological improvement in a mean of 5 months (range, 3-7) after GKR.

\section{A representative case of radiation necrosis after GKR}

A 71-year-old female patient had a $1.5 \mathrm{~cm}$ enhancing mass with perilesional edema on the left cingulate gyrus (Fig. 3A). This was an asymptomatic, metachronous metastatic tumor from an adenocarcinoma in the lung. GKR was administered for a tumor volume of $0.7539 \mathrm{~mL}$ with a marginal prescription dose of 24 Gy. The prescription isodose was 50\%. After GKR, targeted therapy was administered. One and half months after GKS, the enhancing mass had increased and perilesional edema became aggravated without neurological symptoms (Fig. $3 \mathrm{~B}$ and $\mathrm{C})$. Radiation necrosis was diagnosed, and radiologic follow-up was done without any medical treatment. Seven months after GKR, follow-up brain MRI showed the stable mass in size with improved perilesional edema (Fig. 3D).
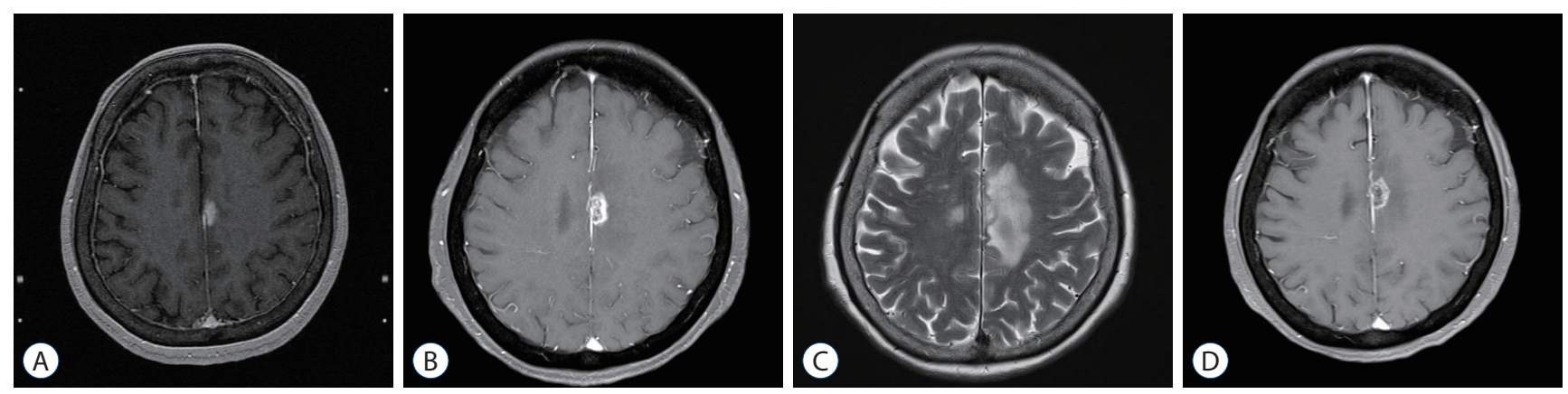

Fig. 3. A representative case with radiation necrosis after gamma knife radiosurgery (GKR). A : A $1.5 \mathrm{~cm}$-sized heterogeneously enhancing mass in the left cingulate gyrus with perilesional edema on T1-weighted magnetic resonance imaging (MRI) with gadolinium enhancement. B : One and half months after GKR, a slightly increase in the size of the enhancing mass $(1.6 \mathrm{~cm})$ was observed on T1-weighted MRI with gadolinium enhancement. C: One and half months after GKR, brain T2-weighted MRI showed aggravated perilesional edema. D : Seven months after GKR, follow-up brain MRI showed a decrease in size of the enhancing mass and perilesional edema. 


\section{DISCUSSION}

GKR has been known to be an effective non-invasive treatment for controlling brain metastases from lung cancer, with 81-98\% local tumor control rates; however, the reported local control rates of GKR vary depending on primary cancer ${ }^{6,14)}$. Moreover, GKS has a low rate of adverse effects such as cognitive impairment, especially in elderly patients, and other radiation toxicities compared to $\mathrm{WBRT}^{8,10,16)}$. It has been previously reported that GKR prescription doses for brain metastases can range from 15-25 Gy in a single irradiation, adjusted according to the tumor volume, primary tumor pathology, and previous brain treatment ${ }^{8,31)}$. The most important factor in determining the GKR prescription radiation doses is tumor volume. Increasing the radiation dose is associated with an increased risk of radiation necrosis, while lowering the dose might decrease the tumor local control effect. Hence, there is no consensus about the appropriate prescription dose.

In 2000, Shaw et al. ${ }^{23)}$ (RTOG 90-05) reviewed the maximum tolerated doses for non-brainstem metastases treated with stereotactic radiosurgery for small to medium-sized tumors $<21 \mathrm{~mm}, 21-30 \mathrm{~mm}$, and $31-40 \mathrm{~mm}$ in diameter. The maximum tolerated doses were determined as $24 \mathrm{~Gy}, 18 \mathrm{~Gy}$, and 15 Gy for metastatic lesions $<21 \mathrm{~mm}, 21-30 \mathrm{~mm}$, and 31$40 \mathrm{~mm}$, respectively. According to this study, small to medium-sized $(<2 \mathrm{~cm}$ in diameter, volume $<5 \mathrm{~mL}$ ) metastases can be safely and effectively treated with a prescription dose of 24 Gy. On exceeding the maximum tolerated dose, severe neurological symptoms that required steroid treatment were observed in $10 \%$ of the patients, radio-necrosis requiring surgery in $10 \%$, and fatal radio-necrosis in 3\%. In 2006, Vogelbaum et al. $^{27)}$ reviewed 202 patients with 375 tumors $(2 \mathrm{~cm}$ or less in maximal diameter), which were treated with a prescription dose of 15 to $24 \mathrm{~Gy}$. The observed a local control rate of $85 \%$ in tumors treated with $24 \mathrm{~Gy}$, compared with $49 \%$ in tumors treated with 18 Gy and 45\% in tumors treated with 15 Gy. A prescription dose of $24 \mathrm{~Gy}$ had a significantly better local control rate than did a dose of 15 or $18 \mathrm{~Gy}$, whereas there was no significant difference between the groups that received $15 \mathrm{~Gy}$ and 18 Gy. No cases of acute radiotoxicity were seen, and only a $10 \%$ incidence of chronic toxicity was observed. In 2017, Mohammadi et al. ${ }^{18)}$ reported the data of 896 patients with 3034 tumors ( $2 \mathrm{~cm}$ or less), which were treated with GKR. The prescription dose was 15 to $24 \mathrm{~Gy}$. With 6.2 months of median follow-up, the prescription dose of 24 Gy to the median isodose line of $56 \%$ showed significantly decreased rates of local failure compared to that with lower doses (15-23 Gy). The incidence of local failure was $4 \%$ and $5 \%$ for a prescription dose of $24 \mathrm{~Gy}$ and $<24 \mathrm{~Gy}$, respectively. Seven percent of all targeted lesions had radiographic radiation necrosis, of which only $4 \%$ were symptomatic but were in the clinically acceptable range.

In this study, we investigated whether tumor control rate improved when the radiation dose was increased and the prognostic factors that influence tumor control. We found that a small tumor size $(p=0.003)$, high radiation dose $(p=0.014)$, and targeted therapy ( $p=0.004$ ) were associated with good tumor control. When analyzed by tumor volume, small-sized tumors (less than $1 \mathrm{~mL}$ ) showed a trend of longer PFS without symptomatic radiation necrosis when the prescription dose was increased up to $24 \mathrm{~Gy}(p=0.072)$. However, when multivariate analysis was performed, GKR combined targeted therapy was more effective in tumor control than tumor volume and radiation dose, when radiation doses of $18 \mathrm{~Gy}$ or more were administered in small and medium-sized tumors. Therefore, in small-sized metastatic tumors, when the prescription dose was increased up to $24 \mathrm{~Gy}$, tumor control became better without symptomatic radiation necrosis; however, the effect of systemic anti-cancer regimen should also be considered.

GKR uses targeted radiation that spares the normal brain tissue, preserves cognitive function, and alleviates symptoms of brain tumor but causes radiation injury in the white matter resulting in a necrotic lesion, which is the only significant complication of $\mathrm{GKR}^{22)}$. Radiation necrosis is caused by vascular injury, which leads to the expression of vascular endothelial growth factor (VEGF) and breakdown of the $\mathrm{BBB}^{13,17,19,33)}$. High levels of VEGF expression lead to abnormal neovascularization, and the vessels have a fragile structure and exhibit high permeability, which promotes exudation into the surrounding brain parenchyma leading to edema formation. This causes a localized increase in the intracranial pressure, which in turn causes localized ischemic change, resulting in a vicious cycle of localized hypoxia and ultimately to radiation induced brain necrosis $^{13,17,19,33)}$. Therefore, the general strategy for treating radiation necrosis is to reduce the edema, which might lead to neurological adverse effects. The treatment options are high-dose dexamethasone and surgical resection ${ }^{12)}$. The major risk factors for radiation necrosis after stereotactic radiosurgery are tumor volume and radiation dose ${ }^{6}$. In a study by Mo- 
hammadi et al. ${ }^{18)}$ the incidence of radiation necrosis in small to medium-sized tumors (less than $2 \mathrm{~cm}$ in diameter) was $8.5 \%$ when the prescription dose was $24 \mathrm{~Gy}$ and $6.5 \%$ when the dose was $<24$ Gy. In our study, the incidence of radiation necrosis was $2.2 \%$. Nevertheless, in the groups with small and medium-sized brain metastases, radiation necrosis was not severe enough to require surgical resection and resolved with or without steroid therapy for several weeks.

Previously, cytotoxic chemotherapy with a platinum-based first-line drug alone was usually used in patients with NSCLC and brain metastasis; however, the metastatic tumors in the brain could not be controlled, and the disease usually progressed $^{24)}$. Within the last decade, significant advances in molecular pathology have provided a better understanding of the underlying pathology of NSCLC, leading to the development of new therapies such as molecular targeted therapy ${ }^{5}$. In some recent papers, it has been reported that targeted therapy with small molecules such as tyrosine kinase inhibitor is effective in treating brain lesions; these small molecules can penetrate the BBB and reach CSF concentrations that are $1.5-7 \%$ of their plasma concentrations ${ }^{4,7,32)}$. Hence, the OS of patients with brain metastases from NSCLC is better when targeted therapy is used than when other anti-cancer regimens are used. With the recent development of newer generation of drugs for targeted therapies, the capability of these drugs to penetrate the BBB has increased, and the CNS effect has also increased $^{20,21)}$. The latest targeted agents have shown to be effective for tumor free progression survival included newly brain metastases to both in patients with or without CNS lesions, which may indicate a protective effect of these agents against the development of CNS metastases.

In our study, GKR combined targeted therapy was associated with a significantly longer PFS compared to cytotoxic chemotherapy. The correlation between the duration of targeted therapy and PFS of GKR-treated lesions was also statistically significant. It indicates that GKR combined targeted therapy could be an important factor in tumor control of GKR-treated lesions. It might be that the radiation increased the permeability of $\mathrm{BBB}$, further increasing the effectiveness of targeted therapy ${ }^{11,25,26)}$. Previously mentioned, newer generation drugs have good control rate of primary lung cancer and brain tu$\operatorname{mor}^{20,21)}$. In our study, GKR combined targeted therapy showed significantly better OS in patients and PFS of GKRtreated lesions, but did not affect the occurrence of new le- sions in the brain. These could be due to the use of various generations of targeted agents for a long time. There were 37 patients who used the first-generation drug as gefitinib, erlotinib or crizotinib, nine patients used the second-generation drug such as afatinib and five patients used the third-generation drug as rociletinib, lorlatinib or osimertinib.

For patients who were scheduled for targeted therapy, serial brain imaging follow-up without GKR could be considered for small-sized and asymptomatic brain metastases, and for medium-sized brain metastases, lowering the radiation dose to 18 Gy could be considered to prevent side effects

\section{CONCLUSION}

It is difficult to accurately determine the prescription dose that most efficiently mitigates the risk of recurrence; however, in small-sized metastatic brain tumors, irradiation with up to 24 Gy could achieve better tumor control without severe adverse effects such as symptomatic radiation necrosis. Mediumsized tumors had the potential for developing adverse effects but did not show significant changes in tumor control when the prescription dose was greater than 18 Gy. Furthermore, GKR combined targeted therapy could be an important factor in improving tumor control for GKR-treated lesions.

\section{CONFLICTS OF INTEREST}

No potential conflict of interest relevant to this article was reported.

\section{INFORMED CONSENT}

This type of study does not require informed consent.

\section{AUTHOR CONTRIBUTIONS}

\author{
Conceptualization : IJO, IYK, TYJ \\ Data curation : SJP, SHL, SKK \\ Formal analysis : TYJ \\ Funding acquisition : TYJ
}




\author{
Methodology : SJP \\ Project administration : TYJ, IJO \\ Visualization: SJP \\ Writing - original draft : SJP, TYJ \\ Writing - review \& editing: YJK, SJ, KSM, JHH
}

\section{ORCID}

Sue Jee Park https://orcid.org/0000-0001-9323-1534

Sa-Hoe Lim https://orcid.org/0000-0002-1121-0826

Young-Jin Kim https://orcid.org/0000-0003-0376-6784

Kyung-Sub Moon https://orcid.org/0000-0002-1129-1064

In-Young Kim https://orcid.org/0000-0002-1152-119X

Shin Jung https://orcid.org/0000-0003-2587-1243

Seul-Kee Kim https://orcid.org/0000-0002-1508-5057

In-Jae Oh https://orcid.org/0000-0003-4837-1321

Jong-Hwan Hong https://orcid.org/0000-0002-7864-1145

Tae-Young Jung https://orcid.org/0000-0002-8685-8520

\section{- Acknowledgements}

This study was supported by the Basic Science Research Program through the National Research Foundation of Korea (NRF), funded by the Ministry of Science, ICT, \& Future Planning (2020R1I1A3073338).

\section{References}

1. Abdallah SM, Wong A : Brain metastases in non-small-cell lung cancer: are tyrosine kinase inhibitors and checkpoint inhibitors now viable options? Curr Oncol 25(Suppl 1) : S103-S114, 2018

2. Baschnagel AM, Meyer KD, Chen PY, Krauss DJ, Olson RE, Pieper DR, et al. : Tumor volume as a predictor of survival and local control in patients with brain metastases treated with gamma knife surgery. J Neurosurg 119 : 1139-1144, 2013

3. Blagden SP, Charman SC, Sharples LD, Magee LR, Gilligan D : Performance status score: do patients and their oncologists agree? Br J Cancer 89 : 1022-1027, 2003

4. Broniscer A, Panetta JC, O'Shaughnessy M, Fraga C, Bai F, Krasin MJ, et al. : Plasma and cerebrospinal fluid pharmacokinetics of erlotinib and its active metabolite OSI-420. Clin Cancer Res 13 : 1511-1515, 2007

5. Chan BA, Hughes BG : Targeted therapy for non-small cell lung cancer: current standards and the promise of the future. Transl Lung Cancer Res 4 : 36-54, 2015
6. Chin $L S, M a L$, DiBiase $S$ : Radiation necrosis following gamma knife surgery: a case-controlled comparison of treatment parameters and longterm clinical follow up. J Neurosurg 94 : 899-904, 2001

7. Fan Y, Huang Z, Fang L, Miu L, Lin N, Gong L, et al. : Chemotherapy and EGFR tyrosine kinase inhibitors for treatment of brain metastases from non-small-cell lung cancer: survival analysis in 210 patients. Onco Targets Ther 6 : 1789-1803, 2013

8. Frischer JM, Fraller A, Mallouhi A, Vogl UM, Baier F, Ertl A, et al. : Evaluation of dose-staged gamma knife radiosurgical treatment method for high-risk brain metastases. World Neurosurg 94 : 352-359, 2016

9. Gaspar L, Scott C, Rotman M, Asbell S, Phillips T, Wasserman T, et al. : Recursive partitioning analysis (RPA) of prognostic factors in three Radiation Therapy Oncology Group (RTOG) brain metastases trials. Int J Radiat Oncol Biol Phys 37 : 745-751, 1997

10. Higuchi Y, Serizawa T, Nagano O, Matsuda S, Ono J, Sato M, et al. : Three-staged stereotactic radiotherapy without whole brain irradiation for large metastatic brain tumors. Int J Radiat Oncol Biol Phys 74 : 1543-1548, 2009

11. Hochmair $\mathrm{M}$ : Medical treatment options for patients with epidermal growth factor receptor mutation-positive non-small cell lung cancer suffering from brain metastases and/or leptomeningeal disease. Target Oncol 13 : 269-285, 2018

12. Jung EW, Choi J, Chao ST, Murphy ES, Suh JH : Chapter 8 - Principles and Tenets of Radiation Treatment in Glioblastoma in Brem S, Abdullah KG (eds) : Glioblastoma, ed 1. Amsterdam : Elsevier, 2016, pp105-132

13. Kamiryo T, Lopes MB, Kassell NF, Steiner L, Lee KS : Radiosurgeryinduced microvascular alterations precede necrosis of the brain neuropil. Neurosurgery 49 : 409-414; discussion 414-415, 2001

14. Kim KH, Lee MH, Cho KR, Choi JW, Kong DS, Seol HJ, et al. : The influence of histology on the response of brain metastases to gamma knife radiosurgery: a propensity score-matched study. Acta Neurochir (Wien) $160:$ 2379-2386, 2018

15. Lin NU, Lee EQ, Aoyama H, Barani IJ, Barboriak DP, Baumert BG, et al. : Response assessment criteria for brain metastases: proposal from the RANO group. Lancet Oncol 16 : e270-e278, 2015

16. Mekhail T, Sombeck M, Sollaccio R : Adjuvant whole-brain radiotherapy versus observation after radiosurgery or surgical resection of one to three cerebral metastases: results of the EORTC 22952-26001 study. Curr Oncol Rep 13 : 255-258, 2011

17. Miyatake S, Nonoguchi N, Furuse M, Yoritsune E, Miyata T, Kawabata S, et al. : Pathophysiology, diagnosis, and treatment of radiation necrosis in the brain. Neurol Med Chir (Tokyo) 55 : 50-59, 2015

18. Mohammadi AM, Schroeder JL, Angelov L, Chao ST, Murphy ES, Yu JS, et al. : Impact of the radiosurgery prescription dose on the local control of small ( $2 \mathrm{~cm}$ or smaller) brain metastases. J Neurosurg 126 : 735 743, 2017

19. Nonoguchi N, Miyatake S, Fukumoto M, Furuse M, Hiramatsu R, Kawabata $\mathrm{S}$, et al. : The distribution of vascular endothelial growth factorproducing cells in clinical radiation necrosis of the brain: pathological consideration of their potential roles. J Neurooncol 105 : 423-431, 2011 
20. Peters S, Camidge DR, Shaw AT, Gadgeel S, Ahn JS, Kim DW, et al. : Alectinib versus crizotinib in untreated ALK-positive non-small-cell lung cancer. N Engl J Med 377 : 829-838, 2017

21. Reungwetwattana T, Nakagawa $K$, Cho BC, Cobo M, Cho EK, Bertolini A, et al. : CNS response to osimertinib versus standard epidermal growth factor receptor tyrosine kinase inhibitors in patients with untreated EGFR-mutated advanced non-small-cell lung cancer. J Clin Oncol 36 : 33, 3290-3297, 2018

22. Schimmel WCM, Verhaak E, Hanssens PEJ, Gehring K, Sitskoorn MM : A randomised trial to compare cognitive outcome after gamma knife radiosurgery versus whole brain radiation therapy in patients with multiple brain metastases: research protocol CAR-study B. BMC Cancer 18 : 218, 2018

23. Shaw E, Scott C, Souhami L, Dinapoli R, Kline R, Loeffler J, et al. : Single dose radiosurgical treatment of recurrent previously irradiated primary brain tumors and brain metastases: final report of RTOG protocol 9005. Int J Radiat Oncol Biol Phys $47:$ 291-298, 2000

24. Sun YW, Xu J, Zhou J, Liu WJ : Targeted drugs for systemic therapy of lung cancer with brain metastases. Oncotarget $9:$ 5459-5472, 2018

25. Trnovec T, Kállay Z, Bezek $S$ : Effects of ionizing radiation on the blood brain barrier permeability to pharmacologically active substances. Int J Radiat Oncol Biol Phys 19 : 1581-1587, 1990

26. van Vulpen M, Kal HB, Taphoorn MJ, El-Sharouni SY : Changes in blood- brain barrier permeability induced by radiotherapy: implications for timing of chemotherapy? (Review). Oncol Rep 9 : 683-688, 2002

27. Vogelbaum MA, Angelov L, Lee SY, Li L, Barnett GH, Suh JH : Local control of brain metastases by stereotactic radiosurgery in relation to dose to the tumor margin. J Neurosurg $104:$ 907-912, 2006

28. Wrona A, Dziadziuszko R, Jassem J : Management of brain metastases in non-small cell lung cancer in the era of tyrosine kinase inhibitors. Cancer Treat Rev 71 : 59-67, 2018

29. Yamamoto M, Serizawa T, Shuto T, Akabane A, Higuchi Y, Kawagishi J, et al. : Stereotactic radiosurgery for patients with multiple brain metastases (JLGK0901): a multi-institutional prospective observational study. Lancet Oncol 15 : 387-395, 2014

30. Yomo S, Hayashi M : A minimally invasive treatment option for large metastatic brain tumors: long-term results of two-session Gamma knife stereotactic radiosurgery. Radiat Oncol 9 : 132, 2014

31. Yu JB, Schulder $M$, Knisely J : Radiosurgical dose selection for brain metastasis. Prog Neurol Surg 25 : 139-147, 2012

32. Zhao J, Chen M, Zhong W, Zhang L, Li L, Xiao Y, et al. : Cerebrospinal fluid concentrations of gefitinib in patients with lung adenocarcinoma. Clin Lung Cancer 14 : 188-193, 2013

33. Zhuang H, Shi S, Yuan Z, Chang JY : Bevacizumab treatment for radiation brain necrosis: mechanism, efficacy and issues. Mol Cancer 18 : 21, 2019 